\title{
The Dutch Technical-Tactical Tennis Test (D4T) for Talent Identification and Development: Psychometric Characteristics
}

\author{
by \\ Nikki Kolman', Barbara Huijgen ${ }^{1}$, Tamara Kramer1,2, Marije Elferink-Gemser ${ }^{1}$, \\ Chris Visscher ${ }^{1}$
}

This study examined the test-retest reliability, validity and feasibility of the newly developed Dutch TechnicalTactical Tennis Test (D4T). This new test is relevant for talent identification and development in tennis. Thirty-two youth male tennis players (age $13.4 \pm 0.5)$ were classified as elite $(n=15)$ or sub-elite $(n=17)$ according to their position on the national youth ranking list under 15 years (cut-off rank 50) in the Netherlands. Games, rallies and different tactical situations (i.e. offensive, neutral and defensive) were simulated with a ball machine. Players had to return 72 balls to predetermined target areas. Stroke quality was recorded based on ball velocity and accuracy (VAindex), as well as percentage errors. Test-retest reliability was assessed by comparing differences between the first and second test-session $(n=10)$. An intraclass-correlation coefficient of .78 for the VA-index was found $(p<.05)$, indicating excellent test-retest reliability. Independent $t$-tests revealed that elite players outscored sub-elite players for the VAindex, ball velocity, accuracy and percentage errors $(p<.05)$, supporting good validity. Furthermore, a high correlation was found between the VA-index and individual positions on the youth ranking list $(r=-.75 ; p<.001)$. The assessment of feasibility indicated that the D4T was applicable for instructors and coaches. In conclusion, the D4T was shown to be a reliable, valid and feasible test to measure technical-tactical characteristics of tennis performance in youth players.

Key words: racquet sports, field test, performance, ball velocity, accuracy, youth sports.

\section{Introduction}

In tennis especially technical and tactical characteristics are crucial for performance (MacCurdy, 2006; Meylan et al., 2010). Technique in tennis is generally characterized by stroke quality of which the key determinants are the combination of ball velocity and accuracy along with the success rate (Landlinger et al., 2012; Vergauwen et al., 2004). A clear relationship between ball velocity and the level of tennis performance exists, with international players producing higher ball velocities than national players (Landlinger et al., 2012; Martin et al.,
2014). Also a relationship between ball accuracy and the level of tennis performance has been shown, although up to now exclusively between experts and non-experts (Lyons et al., 2013) and not between international and national players, with the exception of lateral stroke accuracy (Landlinger et al., 2012). To our knowledge, only one study has measured ball velocity as well as ball accuracy in youth tennis players (Vergauwen et al., 2004). In this study, a rally test was conducted on a court with reduced dimensions $(13.40 \times 6.10 \mathrm{~m})$ to measure groundstroke

1 - Center for Human Movement Sciences, University Medical Center Groningen, University of Groningen, The Netherlands.

2 - HAN Sports and Exercise, HAN University of Applied Sciences, the Netherlands; Center for Humam Movement Sciences, University Medical Center Groningen, University of Groningen, the Netherlands. 
performance in youth players (age: $12.7 \pm 0.7 \mathrm{yrs}$ ). The results revealed that players with more experience generated a higher success rate, ball velocity and ball accuracy than their less experienced counterparts.

Technical characteristics are needed for the execution of appropriate tactics in a given situation. Consequently, when technical characteristics develop, the quality of tactical characteristics can improve as well (Wang et al., 2013). Tactical characteristics are defined as the knowledge about in-game adaptations and decision-making activity on the court (ElferinkGemser et al., 2010). Anticipation of actions of an opponent together with making appropriate decisions are important aspects of a successful tactical performance. Players have to deal with these aspects instantly (Balser et al., 2014; Féry and Crognier, 2001). Expert players have more experience in performing sport-specific actions and anticipating their opponents' actions than novices (Williams et al., 2002). Williams et al. (2002) showed that expert players anticipated better on their opponents and made earlier decisions than novices, which is a substantial advantage for the execution of groundstrokes. Furthermore, Crognier and Féry (2005) showed that the anticipation of strokes was dependent of the tactical situation (i.e. offensive, neutral and defensive). They indicated that players' accuracy in anticipating the direction in which to move to intercept the ball was close to $80 \%$ when the players were in the offensive situation, while their accuracy was lower when they were in a defensive situation.

To measure performance characteristics in tennis, field tests have been used (Landlinger et al., 2012; Lyons et al., 2013; Vergauwen et al., 1998, 2004). An example of a test that measures technical and tactical characteristics is the Leuven Tennis Performance Test (LTPT) (Vergauwen et al., 1998). In this test, players have to direct their strokes to a target point located at the intersection of the baseline and the sideline. In the LTPT, the higher ranked players made fewer errors than their lower ranked counterparts. Furthermore, they scored higher on ball velocity and lateral stroke accuracy than lower ranked players. However, in this test the risk of the ball landing outside the court is high (Landlinger et al., 2012) and it should be noted that a higher risk resulting in more errors is detrimental for the success rate of strokes.

Previous research in youth tennis showed that on average a rally was between 2.5 and 3 strokes, with more strokes per rally on slow than on fast surfaces (Fernandez-Fernandez et al., 2009). Moreover, data recorded from 481 matches on grass revealed that the average number of rallies per game was approximately six (Magnus and Klaassen, 1999). Therefore, to be realistic with match play, a new field test should include three strokes per rally, six rallies per game and a target area that is located inside the court. At the moment, no reliable and valid test exists that includes the aforementioned aspects and measures technical as well as tactical characteristics in youth tennis players.

The overall purpose of the current study was to develop a reliable, valid and feasible technical-tactical test that could be used in talent identification and development in tennis. It was investigated whether the test was reliable and whether differences in position on the youth ranking list would be manifested in the test performance, supporting its validity. Moreover, it was analyzed whether anticipation and decisionmaking influenced the execution of groundstrokes differently in players with different positions on the ranking list. Finally, it was evaluated whether the test could be applicable for instructors and coaches.

\section{Material and Methods}

\section{Participants}

Thirty-two youth male players (age $13.4 \pm$ 0.5 ; body height $167.7 \pm 10.6 \mathrm{~cm}$; body mass $52.3 \pm$ $10.9 \mathrm{~kg}$ ) were recruited via tennis organizations, clubs and coaches who all gave permission for this study. Participants were classified as elite $(\mathrm{n}=$ $15)$ or sub-elite $(n=17)$ according to their position on the Dutch national youth ranking list under 15 years at the time of testing (KNLTB). Elite players were those ranked among the top-50, while subelite players were classified between position 51 and 350 on the ranking list. Test-retest reliability was assessed in ten sub-elite players.

\section{Measures}

The Dutch Technical-Tactical Tennis Test (D4T) consisted of 72 strokes, grouped in four games of six rallies, in which each rally included three strokes. A ball machine (Pro Match 
Smartshot, Mubo, Gorinchem, Netherlands) was used to meet the criterion of standardization and to feed balls to the participants. Ball accuracy was measured using target areas to which participants were instructed to direct their strokes. A large target area $(3.6 \times 2.7 \mathrm{~m})$ and a middle target area $(2.4 \times 1.8 \mathrm{~m})$ were located at both corners at the intersection of the baseline and the sideline as displayed in Picture 1. A small target area (1.2 $\times$ $0.9 \mathrm{~m}$ ) was located inside the middle target area and was positioned $0.45 \mathrm{~m}$ from the sideline and $0.6 \mathrm{~m}$ from the baseline. The colored target areas were stitched on a large carpet and a colored cap was placed in the center of the small target area as the main target point.

Each game was divided into two offensive, two neutral and two defensive rallies, representing different tactical situations as displayed in Picture 2. Offensive rallies consisted of three ball projections just beyond the service line. Neutral rallies comprised three ball projections to the area around the middle of the court approximately one meter before the baseline, and defensive rallies included three ball projections to the sideline and beyond the service line. The velocity of the ball projections was approximately 70,75 and $80 \mathrm{~km} / \mathrm{h}$, in the offensive, neutral and defensive rallies, respectively. The time interval between the ball projections was $2.5 \mathrm{~s}$ in each tactical situation.

The order of the tactical situations (i.e. offensive, neutral and defensive) in games occurred randomly. However, the order of the games during the test was the same for each participant, since the degree of difficulty was increased during successive games. In the first game, during each rally, participants had to return their strokes to the left target area (deuce side). In the second game, they had to direct the strokes to the right target area (advantage side) and finally, in the third game, participants had to aim their strokes alternately between the two target areas. For example, if a participant directed the strokes in one rally to the left/right/left target area, in the next rally they had to aim their strokes to the right/left/right target area. In the final game, the target area was determined by lights which turned red either on the left or right side of the court. The lights were positioned on tripods and placed in both corners of the singles court just behind the baseline and after the target areas. A light gate was placed before the ball machine. After a ball passed the light gate, a signal was sent by an interface and computer to the lights with an adjustable delay set at $500 \mathrm{~ms}$. Following a prescribed protocol and after the signal was given, one of the two lights turned red. The lights were illustrative of the position of an (artificial) opponent. Hence, participants had to return their strokes to the opposite side of the red light. The complete test design is displayed in Picture 2.

\section{Ball velocity and accuracy}

Ball velocity was measured using a radar system (Ball coach pocket radar, PR1000-BC) and was recorded after each stroke. In the current study, a high degree of reliability was found between the velocity of the radar system and the velocity calculated using captured video images. The single measure intra-class correlation coefficient (ICC) was .988 with a $95 \%$ confidence interval from .979 to $.993, \mathrm{~F}(1,49)=162.303, p<$ .001. Ball accuracy was determined by recording the landing position of the ball using two HDcameras (JVC Everio GZ-GX1). A total of nine, six and three points were awarded to balls landing inside the small, middle and large target area, respectively, as displayed in Picture 1. When balls landed outside the target areas, although still in the court on the correct side (determined by a given game situation), one point was awarded. When balls landed in the wrong side of the court, outside the singles lines or in the net, zero points were awarded.

A measure of stroke quality, the velocityaccuracy-index (VA-index) was calculated by the following formula wherein velocity was expressed in $\mathrm{km}$ per hour (kph):

Velocity - accuracy index $=\frac{k p h^{2}}{100} \times \frac{(\text { sum of achieved points })}{(\text { number of strokes } \times 9)}$

Due to the nonlinear transformation of ball velocity, the higher the ball velocity, the more a given increment in ball velocity was rewarded, thus the velocity in the formula was squared $\left(\mathrm{kph}^{2}\right)$ (Vergauwen et al., 1998). The sum of achieved points was described as the amount of points given to balls landing inside the target areas, the number of strokes was defined as the number of strokes in a particular game, tactical situation or complete test. 


\section{Procedures}

The study was approved by the ethical committee of the Medical Faculty of the University of Groningen (Groningen, Netherlands, November 19th, 2015) and was consistent with the ethical requirements for human experimentation in accordance with the Declaration of Helsinki. Experimental procedures and potential risks were explained to parents/legal guardians and participants, who all signed a written informed consent form. Two observers measured the players height and sitting height to the nearest $\mathrm{mm}$, and body mass to the nearest $0.1 \mathrm{~kg}$. On testing days, participants performed a $10 \mathrm{~min}$ warm-up, including $5 \mathrm{~min}$ of groundstrokes. Afterwards, they were alternately tested on an indoor tennis court. In the meantime, the remaining participants conducted a training session at low intensity. During the test, participants were allowed to rest for $20 \mathrm{~s}$ in between the rallies and $90 \mathrm{~s}$ after three games, which was similar to match play (ITF Tennis). Participants were not limited to a particular stance or grip, but were requested to return balls at their own style (except the use of slice strokes) and match pace. Participants were allowed to use their own racket during the test. Before testing, they played four rallies, one of each different tactical situation (i.e. offensive, neutral and defensive) and one rally with the lights, to get accustomed to the test situation. Test-retest reliability was assessed by ten participants who performed the protocol twice within 14 days.

\section{Statistical Analyses}

IBM SPSS Statistics 23 (IBM Corp. Somers, NY) was used for the statistical analyses. Scores on the VA-index, ball velocity, accuracy and percentage errors were checked for normality by exploring normality plots and z-scores for skewness and kurtosis. If values were missing, maximum likelihood estimation was used as substitution method in the missing value analysis. In total $2.1 \%$ of the values of the velocity variable were missing. For the interrater reliability, the Cohen's kappa was used by evaluating the landing position of balls in one session by two observers. The relative and absolute reliability of the D4T were examined using an ICC and a paired $\mathrm{t}$-test, respectively. ICC values were interpreted as poor if $<.40$, good if ranging between .40 and .75 , and excellent if $>.75$, in accordance with the reliability level scale suggested by Fleiss (1986). Measures of agreement were determined by the standard error of measurement (SEM), smallest detectable differences (SDD) and a Bland-Altman plot (De Vet et al., 2006; Weir, 2005). The discriminant validity was evaluated by comparing the scores on the VA-index, ball velocity, accuracy and percentage errors of the elite and sub-elite players using independent t-tests. Moreover, two univariate ANOVAs were executed with the performance level as a between-subject factor and the game number $(1,2,3$, and 4$)$ and tactical situation (offensive, neutral and defensive) as within-subject factors for the dependent variable VA-index. These ANOVAs were performed separately, as the VA-index per game number and tactical situation were distinct outcomes which were not possible to analyze together. The Bonferroni post-hoc test was performed when the main effect of the game number or tactical situation on the performance was significant. The concurrent validity was investigated by correlating the VA-index with the national ranking using the Spearman's correlation coefficient; $p$-values lower than $5 \%$ were considered statistically significant. Finally, the practical feasibility of the D4T was evaluated, although no fixed requirements for feasibility existed (Bowen et al., 2009). Examples of test criteria for good feasibility are such as that the duration of the test should not take too long, the test should be easy to perform by participants and the number of used materials and test leaders should be considerably small.

\section{Results}

\section{Reliability}

For the interrater reliability, the Cohen's kappa revealed an almost perfect agreement between the two observers for the evaluation of the landing position of the balls, $\kappa=.98(p<.001)$. The results for the test-retest reliability of ten participants showed good relative as well as absolute reliability as presented in Table 1. Figure 1 presents the Bland-Altman plot of the test-retest reliability of the VA-index. The plot shows the difference in the VA-index between the first and the second test-session, a mean difference in the VA-index closer to 0 represents a more reliable measure. 


\section{Discriminant validity}

Tables 2 and 3 illustrate the mean scores for the VA-index, velocity, accuracy, and percentage errors for the elite and sub-elite players in the D4T in total, and in the different games and tactical situations, respectively. The elite players scored significantly higher than the sub-elite ones on all variables. No differences between the games for the VA-index were found $(\mathrm{F}(3)=.374, p>.05)$, and also the interaction between the level of performance and the game number (Level $\times$ Game number) for the VA-index was non-significant $(p>.05)$. In contrast, differences were found between tactical situations on the VA-index $(\mathrm{F}(2)=9.293, p<.001)$, players in the offensive situation scored higher on the VAindex than in the defensive situation $(p<.001)$, but not in the neutral situation $(p>.05)$. No differences were found between the VA-index for players in the neutral and defensive situation and also the interaction between the level of performance and tactical situation (Level $\times$ Tactical) was non-significant $(p>.05)$.

\section{Concurrent validity}

The validity for the relation between the VA-index and individual positions on the youth ranking list showed a highly significant Spearman's correlation coefficient of $-.750(p<$ .001 ) as displayed in Figure 2. This means that the players with a high position on the national ranking list tended to have a higher VA-index. The proportion of explained variance was .64, which means that $64 \%$ of the variance in the VAindex was explained by the ranking.

\section{Feasibility}

The maximum time required to prepare the D4T was approximately $15 \mathrm{~min}$ and the mean time required to give clear instructions about the D4T to participants was three minutes. The instructions about the D4T were easy to comprehend. The duration of the D4T was approximately $12 \mathrm{~min}$. The physical demand of the D4T was not very high. In general, the average time required to prepare and administer the D4T to a group of eight participants was three hours. The used materials in the D4T consisted of a ball machine, a radar and light system, cameras and target areas stitched on a carpet. The minimum number of test leaders required to administer the D4T was two.

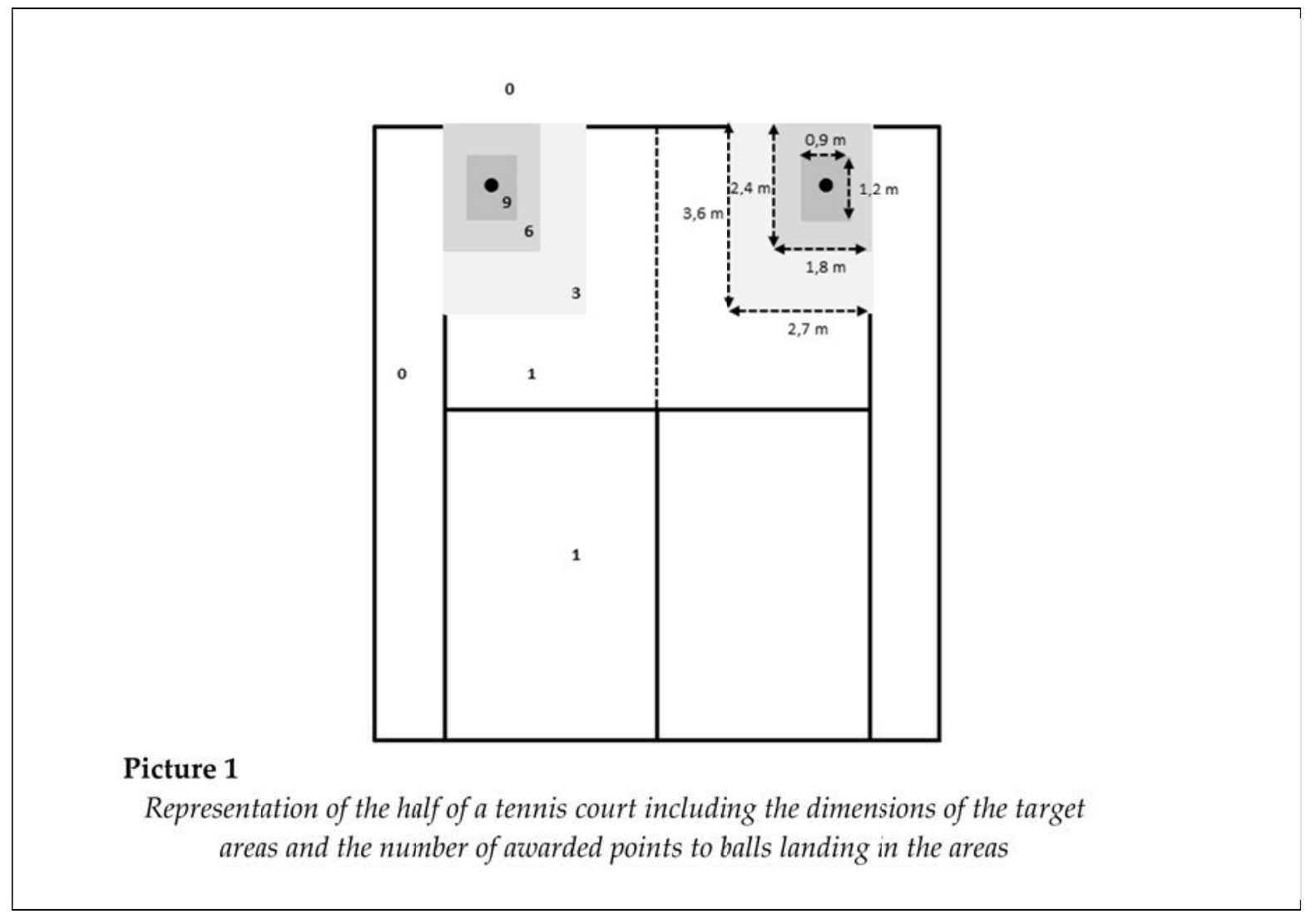




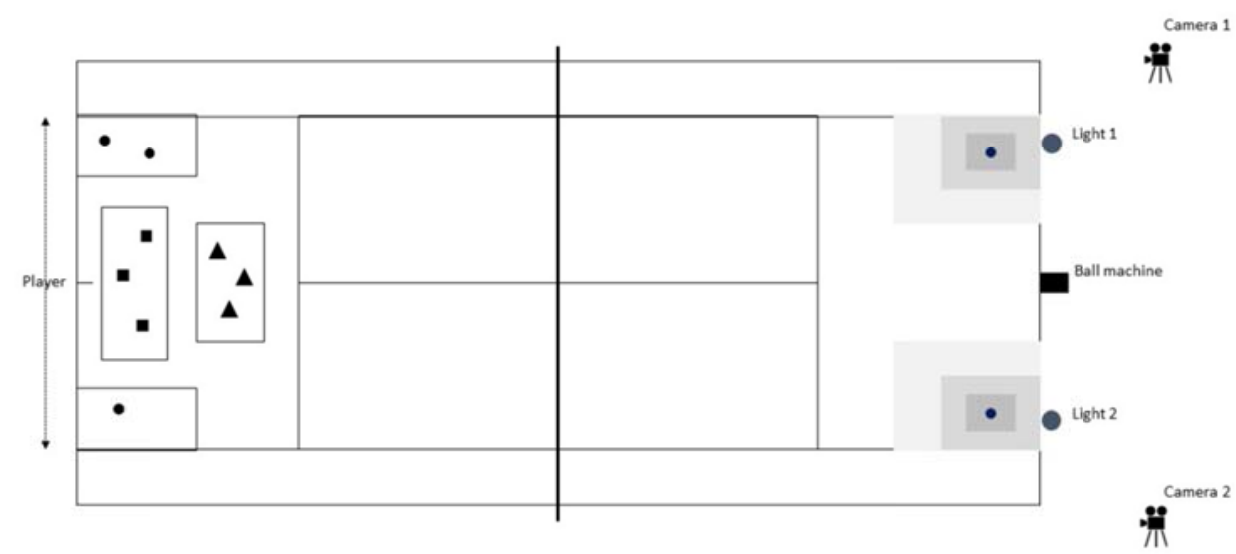

Picture 2.

Representation of the ( $(\boldsymbol{\Delta})$ offensive, $(\boldsymbol{\bullet})$ neutral and $(\bullet)$ defensive tactical situation and the complete test design. The forms represent the three ball projections in the tactical situations.

Table 1

Reliability outcomes of the Dutch Technical-Tactical Tennis Test (D4T) in youth tennis players $(n=10$

\begin{tabular}{|c|c|c|c|c|c|c|c|c|}
\hline & \multicolumn{2}{|c|}{ Mean \pm SD } & \multirow[b]{2}{*}{ ICC } & \multirow{2}{*}{$\begin{array}{c}\text { ICC } \\
95 \% \mathrm{CI}\end{array}$} & \multirow{2}{*}{$\begin{array}{l}\text { Absolute } \\
\text { reliability }\end{array}$} & \multirow{2}{*}{$\begin{array}{c}\text { Absolute } \\
\text { reliability } \\
95 \% \text { CI }\end{array}$} & \multirow[b]{2}{*}{ SEM } & \multirow[b]{2}{*}{ SDD } \\
\hline & $\mathrm{T} 1$ & $\mathrm{~T} 2$ & & & & & & \\
\hline VA-index & $17.92 \pm 3.09$ & $17.63 \pm 4.31$ & $.78^{*}$ & $.19-.95$ & .29 & $-2.06-2.64$ & 2.32 & 6.44 \\
\hline Velocity (kph) & $95.91 \pm 5.43$ & $93.52 \pm 5.86$ & $.87^{* *}$ & $.51-.97$ & $2.40^{*}$ & $.09-4.71$ & 2.28 & 6.33 \\
\hline $\begin{array}{c}\text { Accuracy } \\
\text { (points) }\end{array}$ & $1.75 \pm .29$ & $1.80 \pm .43$ & $.73^{*}$ & $-.02-.93$ & -.05 & $-.30-.20$ & .25 & .69 \\
\hline
\end{tabular}

CI: Confidence Interval; ICC: intra-class correlation coefficient (model: one-way random); SDD: smallest detectable difference; SEM: standard error of measurement; T1: measurement 1; T2: measurement 2 ; ${ }^{*} p<$ $.05 ;{ }^{* *} p<.005$

Table 2

Descriptive statistics (mean $\pm S D$ ) and differences between elite and sub-elite youth tennis players in the D4T $(n=32)$

\begin{tabular}{cccccc}
\hline & $\begin{array}{c}\text { Elite } \\
(\mathrm{n}=15)\end{array}$ & $\begin{array}{c}\text { Sub-elite } \\
(\mathrm{n}=17)\end{array}$ & $\begin{array}{c}\text { Total } \\
(\mathrm{n}=32)\end{array}$ & $\begin{array}{c}\text { Level } \\
\mathrm{t} \text {-value }(\mathrm{df})\end{array}$ & $\mathrm{d}^{\mathrm{b}}$ \\
\hline VA-index & $30.53 \pm 5.44$ & $20.86 \pm 7.81$ & $25.40 \pm 8.30$ & $4.010(30)^{* *}$ & 1.437 \\
Mean velocity $(\mathrm{kph})$ & $106.12 \pm 5.31$ & $97.71 \pm 7.48$ & $101.65 \pm 7.73$ & $3.623(30)^{* *}$ & 1.297 \\
Mean accuracy (points) & $2.44 \pm .40$ & $1.92 \pm .55$ & $2.16 \pm .55$ & $3.048(30)^{* *}$ & 1.091 \\
Percentage errors (\%) & $35.22 \pm 4.06$ & $40.78 \pm 9.87$ & $38.17 \pm 8.1$ & $-2.127(21.822)^{*}$ & .736 \\
\hline
\end{tabular}

at-test (one-tailed) for all variables; ' effect size using Cohen's $d,>.2=$ small, $>.5=$ medium, $>.8=$ large, $>1.3=$ very large; ${ }^{*} p<.05 ;{ }^{* *} p<.005$ 
Table 3

Descriptive statistics (mean $\pm S D$ ) and differences between elite and sub-elite youth tennis players in different games and tactical situations $(n=32)$

\begin{tabular}{|c|c|c|c|c|c|}
\hline & \multicolumn{5}{|c|}{ Interaction } \\
\hline & Elite $(n=15)$ & Sub-elite $(\mathrm{n}=17)$ & Total $(\mathrm{n}=32)$ & $\mathrm{F}(\mathrm{df})^{\mathrm{a}}$ & $p^{\mathrm{b}}$ \\
\hline Game situation & & & & $1.045(3)$ & .375 \\
\hline \multicolumn{6}{|l|}{ Game 1} \\
\hline VA-index & $30.73 \pm 7.83$ & $22.69 \pm 12.40$ & $26.46 \pm 11.12$ & & \\
\hline Mean velocity (kph) & $104.66 \pm 5.21$ & $96.40 \pm 8.97$ & $100.27 \pm 8.45$ & & \\
\hline Mean accuracy (points) & $2.53 \pm .65$ & $2.09 \pm .97$ & $2.30 \pm .85$ & & \\
\hline Percentage errors (\%) & $34.07 \pm 9.82$ & $36.59 \pm 15.97$ & $35.41 \pm 13.30$ & & \\
\hline \multicolumn{6}{|l|}{ Game 2} \\
\hline VA-index & $29.38 \pm 11.99$ & $23.47 \pm 10.07$ & $26.24 \pm 11.23$ & & \\
\hline Mean velocity (kph) & $106.23 \pm 5.30$ & $97.65 \pm 8.02$ & $101.67 \pm 8.05$ & & \\
\hline Mean accuracy (points) & $2.33 \pm .89$ & $2.13 \pm .67$ & $2.22 \pm .78$ & & \\
\hline Percentage errors (\%) & $37.78 \pm 13.16$ & $40.19 \pm 13.83$ & $39.06 \pm 13.35$ & & \\
\hline \multicolumn{6}{|l|}{ Game 3} \\
\hline VA-index & $31.52 \pm 6.31$ & $18.17 \pm 6.53$ & $24.42 \pm 9.26$ & & \\
\hline Mean velocity (kph) & $107.43 \pm 4.86$ & $97.87 \pm 7.39$ & $102.35 \pm 7.90$ & & \\
\hline Mean accuracy (points) & $2.46 \pm .47$ & $1.71 \pm .58$ & $2.06 \pm .65$ & & \\
\hline Percentage errors (\%) & $36.65 \pm 10.45$ & $45.09 \pm 16.08$ & $41.14 \pm 14.18$ & & \\
\hline \multicolumn{6}{|l|}{ Game 4} \\
\hline VA-index & $30.57 \pm 8.32$ & $19.13 \pm 8.04$ & $24.49 \pm 9.92$ & & \\
\hline Mean velocity (kph) & $106.18 \pm 6.95$ & $98.92 \pm 7.28$ & $102.32 \pm 7.92$ & & \\
\hline Mean accuracy (points) & $2.44 \pm .64$ & $1.74 \pm .65$ & $2.07 \pm .72$ & & \\
\hline Percentage errors (\%) & $32.22 \pm 9.21$ & $41.17 \pm 12.60$ & $36.98 \pm 11.86$ & & \\
\hline Tactical situation & & & & $.80(2)$ & .452 \\
\hline \multicolumn{6}{|l|}{ Offensive } \\
\hline VA-index & $37.88 \pm 8.91$ & $24.12 \pm 10.35$ & $29.71 \pm 11.84$ & & \\
\hline Mean velocity (kph) & $108.22 \pm 4.40$ & $100.88 \pm 7.55$ & $103.86 \pm 7.35$ & & \\
\hline Mean accuracy (points) & $2.91 \pm .64$ & $2.09 \pm .71$ & $2.42 \pm .78$ & & \\
\hline Percentage errors (\%) & $31.42 \pm 6.28$ & $39.91 \pm 10.78$ & $36.46 \pm 10.03$ & & \\
\hline \multicolumn{6}{|l|}{ Neutral } \\
\hline VA-index & $31.37 \pm 8.36$ & $21.99 \pm 7.93$ & $25.80 \pm 9.24$ & & \\
\hline Mean velocity (kph) & $106.80 \pm 5.49$ & $99.48 \pm 8.37$ & $102.45 \pm 8.11$ & & \\
\hline Mean accuracy (points) & $2.47 \pm .61$ & $1.95 \pm .61$ & $2.16 \pm .65$ & & \\
\hline Percentage errors (\%) & $35.26 \pm 9.43$ & $40.35 \pm 8.90$ & $38.28 \pm 9.32$ & & \\
\hline \multicolumn{6}{|l|}{ Defensive } \\
\hline VA-index & $26.31 \pm 6.21$ & $17.42 \pm 7.21$ & $21.03 \pm 8.05$ & & \\
\hline Mean velocity (kph) & $103.44 \pm 6.37$ & $95.36 \pm 8.27$ & $98.64 \pm 8.46$ & & \\
\hline Mean accuracy (points) & $2.21 \pm .44$ & $1.69 \pm .58$ & $1.90 \pm .58$ & & \\
\hline Percentage errors (\%) & $38.12 \pm 9.75$ & $40.79 \pm 15.25$ & $39.71 \pm 13.17$ & & \\
\hline
\end{tabular}

Note: ${ }^{a} A N O V A$ for the interaction of Level $\times$ Game number, and Level $\times$ Tactical; ${ }^{b} p$-value (two-tailed 


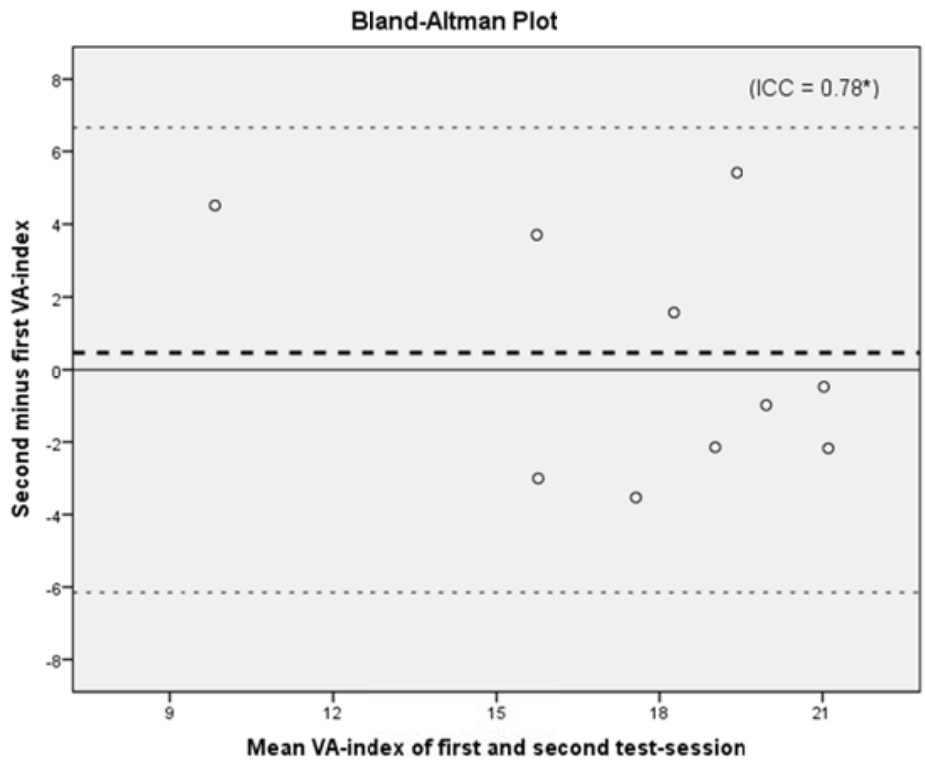

\section{Figure 1}

Bland-Altman plot for the test-retest reliability in youth tennis players $(n=10)$. The bold dotted line represents the difference in the mean VA-index between the first and the second test-session. The non-bold dotted lines represent the $95 \%$ limits of agreement ( \pm $1.96 \times$ SD). ICC: intra-class correlation coefficient (model: one-way random); ${ }^{*} p<.05$.

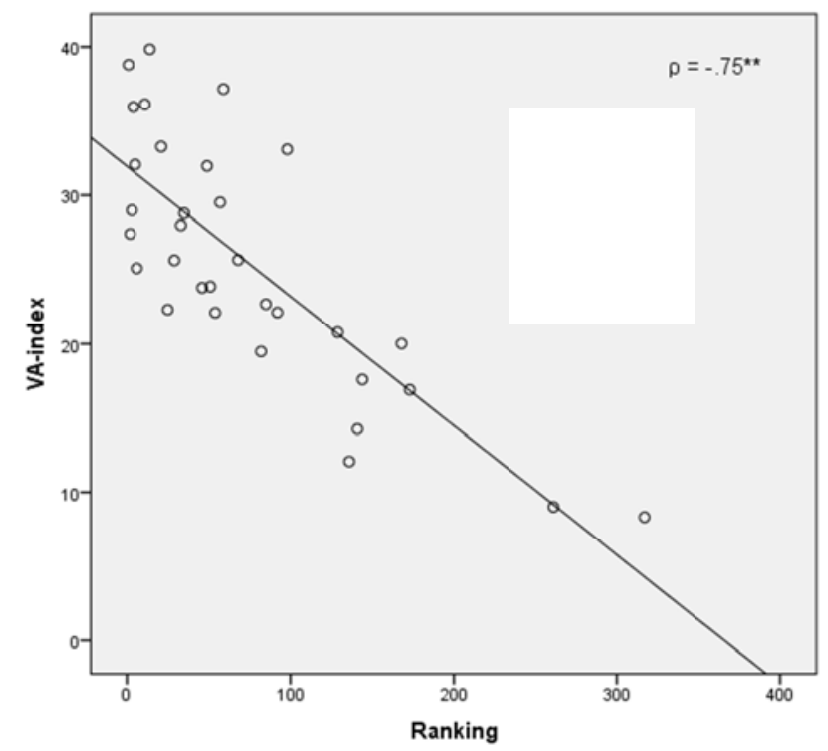

Figure 2

Representation of the relationship between the ranking and the VA-index in youth tennis players $(n=32)$ 


\section{Discussion}

The aim of this study was to develop a reliable, valid and feasible technical-tactical test that could be used for talent identification and development in tennis. The newly developed Dutch Technical-Tactical Tennis Test (D4T) showed good test-retest reliability. Furthermore, the D4T was able to discriminate between elite and sub-elite players; moreover a high correlation was found between individual positions on the youth ranking list and the VA-index, supporting the validity of the test. The assessment of practical feasibility indicated that the D4T was applicable for instructors and coaches.

The results for the interrater reliability showed an almost perfect agreement between two observers for the evaluation of the landing position of the balls. The target areas on the court were recorded with two cameras, thus the landing positions of the balls could be accurately noted, which is an advantage of the D4T. Furthermore, the results for the test-retest reliability indicated that the reliability of the VA-index and ball velocity were excellent, whereas the reliability of the ball accuracy was good in boys aged under 15 . In the current study, test-retest reliability was assessed in the younger sub-elite players, though older elite players have a more consistent tennis performance (McPherson and Thomas, 1989; Vergauwen et al., 2004). Therefore, it is expected that even higher reliability outcomes would be obtained when test-retest reliability is assessed in older and more experienced players. Further research should examine whether the D4T is also reliable in girls and in players in other age categories.

The results revealed that elite players scored higher on the VA-index, ball velocity, accuracy and percentage errors than sub-elite players. This is in line with earlier research that found elite players making less errors and producing higher scores on ball velocity and accuracy than sub-elite players (Landlinger et al., 2012; Lyons et al., 2013; Vergauwen et al., 1998). Furthermore, a strong relationship between individual positions on the national youth ranking list and the VA-index was found. The results showed that players with a high position on the youth ranking list tended to have higher scores on the VA-index; these scores decreased gradually as players had lower positions on the youth ranking list. However, the use of the national ranking list as a measure of performance is a point of discussion. A players' position on the ranking list can be very unstable, because it is partially determined by the number of tournaments played. Since a players' position on the ranking list can be altered in a few weeks, the classification of players as elite or sub-elite can be misleading.

The occurrence of various tactical situations influenced the VA-index, which can be explained by the difference in the degree of difficulty of the projected balls. In the offensive situation, players received the easiest balls, supported by higher scores on the VA-index, compared to lower scores in the more challenging neutral and defensive situation. However, the effect of a tactical situation on performance did not differ between the levels of performance. Contrary to our expectations, the effect of a game situation on performance was non-significant. The results revealed that the performance levels did not vary significantly for the VA-index between the four games. It was expected that differences in the VA-index between performance levels would become more observable in the game with the lights considering that expert players anticipate better and make earlier decisions than novices, which is a substantial advantage for the execution of groundstrokes (Del Villar et al., 2007; McPherson and Thomas, 1989; Williams et al., 2002). However, all players included in the study had enough adequate experience in performing sport-specific actions and dealing with their opponents' actions compared to the novices measured in the aforementioned studies. An alternative explanation might be that the time delay between the ball passing the light gate and the moment of lightning of the signs was too small. In the current protocol, the delay was set at $500 \mathrm{~ms}$, which was possibly sufficient for all players to make appropriate decisions and to execute groundstrokes accurately. Therefore, future research should examine whether the game with the lights is more able to discriminate between particular sports levels when varying the delay.

Concerning the design of the D4T, it was of particular interest to examine the practical feasibility of the test. In general, the average time required to prepare and administer the D4T to a 
group of eight participants was three hours, which is a significant advantage compared to other field tests as it can be easily incorporated in training (Rota et al., 2014; Vergauwen et al., 1998). The materials of the D4T can be reduced for practical usage by instructors and coaches. Usually, a ball machine as well as a radar system to measure ball velocity and cameras to videotape the landing points of the balls are readily available for many sports organizations, instructors and coaches. Furthermore, the carpets with the stitched target areas can be replaced by taping the dimensions of the target areas on the court. Conversely, the requirement for the light system might be more difficult to manage or replace by low-cost alternatives. As mentioned before, future research should analyze various time delays set within the light system. If it appears that the light signals with a different delay do not discriminate between different levels of performance, it should be additionally examined whether the D4T is also reliable and valid without using the light system.

Since the D4T was developed as a test for talent identification and development, it is interesting for future research to investigate whether the test is also able to discriminate between players within the top-50 in the Netherlands. Also, it should be noted that the players in the current study were classified as elite or sub-elite according to the national ranking list in the Netherlands, which cannot be considered as international elite performance level. Therefore, it would be also interesting to include international elite players in future studies using the D4T. The results of the current study revealed that the VAindex was the most distinguishing factor between performance levels, thus, future research should focus on possible differences in the VA-index between players with more homogeneous levels of performance. However, individual scores on ball velocity and accuracy are also essential to be taken into account. The speed-accuracy trade-off hypothesis states that to achieve greater accuracy, the execution time of a movement needs to increase (Fitts, 1954). Earlier research in soccer revealed that elite players were more accurate in their ball control, especially under time pressure, compared to sub-elite players (Huijgen et al., 2013). It would be interesting to further investigate whether talented players are able to maintain accuracy in their strokes when the demands (e.g. speed) of tennis are increased. Furthermore, additional research is needed to indicate if having superior scores on the VA-index during adolescence is indeed a good predictor for future performance in adulthood. However, future research should take into account that, besides technical and tactical characteristics, other factors such as anthropometry, physiology and psychology are important for championship tennis performance. Practical implications for instructors and coaches can be to implement the $\mathrm{D} 4 \mathrm{~T}$ at the beginning and at the end of a season, to monitor players' progress in a season. Differences between the second and the first testsession on the VA-index larger than the SDD of 6.44 are indicative of players' development within a season. In summary, this is the first study that developed a test to measure technical and tactical characteristics in youth tennis players. The D4T was shown to be a reliable, valid and feasible test to assess technical-tactical characteristics of youth tennis players.

\section{Acknowledgements}

The authors would like to thank Nynke Blaauw and Vince Nijboer for their collaboration in the data collection, Wim Kaan for the development of the light system, Winnie Bos for the production of the target areas and the players and coaches for their time and effort in the participation in this study.

\section{References}

Balser N, Lorey B, Pilgramm S, Stark R, Bischoff M, Zentgraf K, Williams M, Munzert J. Prediction of human actions: Expertise and task-related effects on neural activation of the action observation network. Hum Brain Mapp, 2014; 35: 4016-4034 
Bowen DJ, Kreuter M, Spring B, Cofta-Woerpel L, Linnan L, Weiner D, Bakken S, Kaplan CP, Squiers L, Fabrizio C, Fernandez M. How We Design Feasibility Studies. Am J Prev Med, 2009; 36: 452-457

Crognier L, Féry YA. Effect of tactical initiative on predicting passing shots in tennis. Appl Cognitive Psych, 2005; 19: 637-649

Del Villar F, González LG, Iglesias D, Moreno MP, Cervello EM. Expert-novice differences in cognitive and execution skills during tennis competition 1. Percept Motor Skill, 2007; 104: 355-365

De Vet HC, Terwee CB, Knol DL, Bouter LM. When to use agreement versus reliability measures. J Clin Epidemiol, 2006; 59: 1033-1039

Elferink-Gemser MT, Kannekens R, Lyons J, Tromp Y, Visscher C. Knowing what to do and doing it: Differences in self-assessed tactical skills of regional, sub-elite, and elite youth field hockey players. $J$ Sport Sci, 2010; 28: 521-528

Fernandez-Fernandez J, Sanz-Rivas D, Mendez-Villanueva A. A review of the activity profile and physiological demands of tennis match play. Strength Cond J, 2009; 31: 15-26

Féry YA, Crognier L. On the tactical significance of game situations in anticipating ball trajectories in tennis. Res Q Exerc Sport, 2001; 72: 143-149

Fitts PM. The information capacity of the human motor system in controlling the amplitude of movement. J Exp Psychol, 1954; 47: 381-391

Fleiss JL. Reliability of measurement. The Design and Analysis of Clinical Experiments. New York: John Wiley \& Sons, 1-32; 1986

Huijgen BCH, Elferink-Gemser MT, Ali A, Visscher C. Soccer skill development in talented players. Int J Sports Med, 2013; 34: 720-726

Landlinger J, Stöggl T, Lindinger S, Wagner H, Müller E. Differences in ball speed and accuracy of tennis groundstrokes between elite and high-performance players. Eur J Sport Sci, 2012; 12: 301-308

Lyons M, Al-Nakeeb Y, Hankey J, Nevill A. The effect of moderate and high-intensity fatigue on groundstroke accuracy in expert and non-expert tennis players. J Sports Sci Med, 2013; 12: 298-308

MacCurdy D. Talent identification around the world and recommendations for the Chinese Tennis Association, 2006. Available at: http://www.tennis.co.kr/UpLoad/Front/Files/TID_China.pdf; accessed on 12.05.2016

Magnus JR, Klaassen FJ. On the advantage of serving first in a tennis set: four years at Wimbledon. J Roy Stat Soc D-Sta, 1999; 48: 247-256

Martin C, Bideau B, Ropars M, Delamarche P, Kulpa R. Upper limb joint kinetic analysis during tennis serve: Assessment of competitive level on efficiency and injury risks. Scand J Med Sci Spor, 2014; 24: 700-707

McPherson SL, Thomas JR. Relation of knowledge and performance in boys' tennis: Age and expertise. J Exp Child Psychol, 1989; 48: 190-211

Meylan C, Cronin J, Oliver J, Hughes M. Reviews: Talent identification in soccer: The role of maturity status on physical, physiological and technical characteristics. Int J Sports Sci Coach, 2010; 5: 571-592

Rota S, Morel B, Saboul D, Rogowski I, Hautier C. Influence of fatigue on upper limb muscle activity and performance in tennis. J Electromyogr Kinesiol, 2014; 24: 90-97

Vergauwen L, Madou B, Behets D. Authentic evaluation of forehand groundstrokes in young low-to intermediate-level tennis players. Med Sci Sports Exerc, 2004; 36: 2099-2106

Vergauwen L, Spaepen AJ, Lefevre J, Hespel P. Evaluation of stroke performance in tennis. Med Sci Sports Exerc, 1998; 30: 1281-1288

(C) Editorial Committee of Journal of Human Kinetics 
Wang MY, Liu YC, Chen CJ. Techniques and tactics analysis related to personality in table tennis doubles, 2013. Available at: http://ittf.com/ittf_science/SSCenter/docs/Wang\%20M\%20Y_Chen\%20C\%20J-1revised-OK.pdf; accessed on 12.05.2016

Weir JP. Quantifying test-retest reliability using the intraclass correlation coefficient and the SEM. J Strength Cond Res, 2005; 19: 231-240

Williams AM, Ward P, Knowles JM, Smeeton NJ. Anticipation skill in a real-world task: measurement, training, and transfer in tennis. J Exp Psychol Appl, 2002; 8: 259-270

\section{Corresponding author:}

\section{Barbara Huijgen}

Center for Human Movement Sciences, University Medical Center Groningen, University of Groningen.

Antonius Deusinglaan 1, 9713 AV Groningen, the Netherlands

Phone: $+31 / 06 / 25646582$

Fax: + 31/50/363 3150

E-mail: b.c.h.huijgen@umcg.nl 